\title{
'Useful' R-norm Information Measure and its Properties
}

\author{
D.S. Hooda ${ }^{1}$, Keerti Upadhyay ${ }^{2}$ and D.K.Sharma ${ }^{3}$ \\ Jaypee University of Engineering and Technology A.B. Road, Raghogarh - 473226 Distt. Guna - M.P. (India).
}

Abstract : In the present communication, a new 'useful' R-norm information measure has been defined and characterized axiomatically. Its particular cases have been discussed. Properties of the new measure have also been studied.

Keywords: non-additivity, $R$-norm entropy, stochastic independence, utility distribution.

\section{Introduction}

Let us consider the set of positive real numbers, not equal to 1 and denote this by $\mathfrak{R}^{+}$defined as $\mathfrak{R}^{+}=\{R: R \geq 0, R \neq 1\}$. Let $\Delta_{\mathrm{n}}$ with $\mathrm{n} \geq 2$ is the set of all probability distributions

$$
P=\left\{\left(p_{1}, p_{2}, \ldots . ., p_{n}\right), p_{i} \geq 0, \text { for each } i \text { and } \sum_{i=1}^{n} p_{i}=1\right\} \text {. }
$$

[1] studied R-norm information of the distribution P defined for $R \in \mathfrak{R}^{+}$by:

$$
\mathrm{H}_{\mathrm{R}}(\mathrm{P})=\frac{\mathrm{R}}{\mathrm{R}-1}\left[1-\left(\sum_{\mathrm{i}=1}^{\mathrm{n}} \mathrm{p}_{\mathrm{i}}^{\mathrm{R}}\right)^{1 / \mathrm{R}}\right]
$$

The R-norm information measure (1) is a real function $\Delta_{n} \rightarrow \mathfrak{R}^{+}$defined on $\Delta_{n}$, where $\mathrm{n} \geq 2$ and $\mathrm{R}^{+}$is the set of real positive numbers. The measure (1) is different from entropies of [2], [3], [4] and [5]. The main property of this measure is that when $\mathrm{R} \rightarrow 1$ (1) approaches to Shannon's entropy and when $R \rightarrow \infty, H_{R}(P) \rightarrow 1-\max p_{i}$, where $i=1,2, \ldots, n$.

The measure (1) can be generalized in so many ways. [6] Proposed and characterized the following parametric generalization of (1.1):

$H_{R}^{\beta}(P)=\frac{R}{R+\beta-2}\left[1-\left(\sum_{i=1}^{n} p_{i}^{\frac{R}{2-\beta}}\right)^{\frac{2-\beta}{R}}\right], 0<\beta \leq 1, \quad R(>0) \neq 1$

The above measure (2) was called generalized R-norm information measure of degree $\beta$ and it reduces to (1) when $\beta=1$.

Further when $\mathrm{R}=1$ (2) reduces to:

$H_{1}^{\beta}(P)=\frac{1}{\beta-2}\left[1-\left(\sum p_{i}^{\frac{1}{2-\beta}}\right)^{2-\beta}\right], 0<\beta \leq 1$

In case $\gamma=\frac{1}{2-\beta}$ reduces to:

$H^{\gamma}(P)=\frac{\gamma}{\gamma-1}\left[1-\left(\sum_{i=1}^{n} p_{i}^{\gamma}\right)^{\frac{1}{\gamma}}\right], \frac{1}{2}<\gamma \leq 1$

This is an information measure which has been given by [7]. It can be seen that (4) also reduces to Shannon's entropy when $\gamma \rightarrow 1$.

[8] Proposed and studied the following parametric generalization of (1):

$H_{R}^{\alpha, \beta}(P)=\frac{R}{R+\beta-2 \alpha}\left[1-\left(\sum_{i=1}^{n} p_{i}^{\frac{R}{2 \alpha-\beta}}\right)^{\frac{2 \alpha-\beta}{R}}\right], \alpha \geq 1,0<\beta \leq 1, R(>0) \neq 1,0<R+\beta \neq 2 \alpha$ 
They called (5) as the generalized R-norm information measure of type $\alpha$ and degree $\beta$. (5) reduces to (2) when $\alpha=1$ and it further reduces to (1) when $\beta=1$.Recently, [9] have applied (5) in studying the bounds of generalized mean code length.

In order to distinguish the events $E_{1}, E_{2}, \ldots, E_{n}$ with respect to a given qualitative characteristic of physical system taken into account, we ascribe to each event $E_{i}$ a non- negative number $u\left(E_{i}\right)=u_{i}(>0)$ directly proportional to its importance. We call $u_{i}$, the utility or importance of event $E_{i}$ where probability of occurrence is $p_{i}$.In general $u_{i}$ is independent of $p_{i}$ (see [10]).

[11] characterized a quantitative-qualitative measure which was called 'useful' information by [10] of the experiment $\mathrm{E}$ and is given as:

$$
\begin{aligned}
& H(P ; U)=H\left(p_{1}, p_{2}, \ldots, p_{n} ; u_{1}, u_{2}, \ldots, u_{n}\right) \\
& =-\sum u_{i} p_{i} \log p_{i}, \quad u_{i}>0, \quad 0<p_{i} \leq 1, \quad \sum p_{i}=1
\end{aligned}
$$

Later on [12] characterized the following measure of 'useful' information:

$$
H(P ; U)=\frac{-\sum u_{i} p_{i} \log p_{i}}{\sum u_{i} p_{i}}
$$

Analogous to (1) we consider a measure of 'useful' R-norm information as given below:

$$
H_{R}(P ; U)=\frac{R}{R-1}\left[1-\left(\frac{\sum u_{i} p_{i}{ }^{R}}{\sum u_{i} p_{i}}\right)^{1 / R}\right],
$$

where $U=\left(u_{1}, u_{2}, \ldots u_{n}\right)$ is the utility distribution and $u_{i}>0$ is the utility of an event with probability $p_{i}$. It may be noted that if $R \rightarrow 1$, then (8) reduces to (7). Further let $\Delta_{n}^{*}$ be a set of utility distributions s.t. $U \in \Delta_{n}^{*}$ is utility distribution corresponding to $P \in \Delta_{n}$.

In the present paper we characterize the 'useful' R-norm information measure (8) axiomatically in section 2. In section 3 we study the properties of the new measure of 'useful' R-norm information measure.

\section{Axiomatic Characterization}

Let $S_{n}=\Delta_{n} \times \Delta_{n}^{*} \rightarrow R^{+}, \quad n=2,3, \ldots \ldots \ldots$ and $G_{n}$ be a sequence of functions of $p_{i}^{\prime} s$ and $u_{i}^{\prime} s, i=1,2, \ldots \ldots \ldots, n$, defined over $S_{n}$ satisfying the following axioms:

Axiom 2.1. $G_{n}(P: U)=a_{1}+a_{2} \sum_{i=1}^{n} h\left(p_{i}, u_{i}\right)$, where $a_{1}$ and $a_{2}$ are non zero constants, and

$p, u \in J=\{(0,1) \times(0, \infty)\} \cup\{(0, y) ; 0 \leq y \leq 1\} \cup\left\{\left(\infty, y^{\prime}\right): 0 \leq y^{\prime} \leq \infty\right\}$.

This axiom is also called sum property.

Axiom 2.2. For $P \in \Delta_{n}, U \in \Delta_{n}^{*}, P^{\prime} \in \Delta_{m}$, and $U^{\prime} \in \Delta_{m}^{*}, G_{m n}$ satisfies the following property:

$G_{m n}\left(P P^{\prime}: U U^{\prime}\right)=G_{n}(P: U)+G_{m}\left(P^{\prime}: U^{\prime}\right)-\frac{1}{a_{1}} G_{n}(P: U) G_{m}\left(P^{\prime}: U^{\prime}\right)$.

Axiom 2.3. $h(p, u)$ is a continuous function of its arguments $\mathrm{p}$ and $\mathrm{u}$.

Axiom 2.4. Let all $p_{i}^{\prime} s$ and $u_{i}^{\prime} s$ are equiprobable and of equal utility of events respectively, then

$G_{n}\left(\frac{1}{n}, \ldots \ldots \ldots, \frac{1}{n}, u, \ldots \ldots \ldots, u\right)=\frac{R}{R-1}\left(1-n^{\frac{1-R}{R}}\right)$, where $n=2,3, \ldots$, and $R(>0) \neq 1$

First of all we prove the following three lemmas to facilitate to prove the main theorem: 
Lemma 2.1. From axiom 2.1 and 2.2, it is very easy to arrive at the following functional equation:

$$
\sum_{i=1}^{n} \sum_{j=1}^{m} h\left(p_{i} p_{j}^{\prime}, u_{i} u_{j}^{\prime}\right)=\left(\frac{-a_{2}}{a_{1}}\right) \sum_{i=1}^{n} h\left(p_{i}, u_{i}\right) \sum_{j=1}^{m} h\left(p_{j}^{\prime}, u_{j}^{\prime}\right)
$$

where $\left(p_{i}, u_{i}\right),\left(p_{j}^{\prime}, u_{j}^{\prime}\right) \in J$ for $i=1,2, \ldots \ldots \ldots ., n$ and $j=1,2, \ldots \ldots \ldots ., m$.

Lemma 2.2. The continuous solution that satisfies (9) is the continuous solution of the functional equation:

$h\left(p p^{\prime}, u u^{\prime}\right)=\left(\frac{-a_{2}}{a_{1}}\right) h(p, u) h\left(p^{\prime}, u^{\prime}\right)$,

Proof: Let $a, b, c, d$ and $a^{\prime}, b^{\prime}, c^{\prime}, d^{\prime}$ be positive integers such that

$1 \leq a^{\prime} \leq a, 1 \leq b^{\prime} \leq b, 1 \leq c \leq c^{\prime}$, and $1 \leq d \leq d^{\prime}$.

Setting $n=a-a^{\prime}+1=c^{\prime}-c+1$ and $m=b-b^{\prime}+1=d^{\prime}-d+1$,

$p_{i}=\frac{1}{a}\left(i=1,2, \ldots, a-a^{\prime}\right), \quad p_{a-a^{\prime}+1}=\frac{a^{\prime}}{a}$,

$u_{i}=\frac{1}{c}\left(i=1,2, \ldots, c^{\prime}-c\right), u_{c^{\prime}-c+1}=\frac{c^{\prime}}{c}$,

$p_{j}^{\prime}=\frac{1}{b}\left(j=1,2, \ldots, b-b^{\prime}\right), \quad p_{b-b^{\prime}+1}^{\prime}=\frac{b^{\prime}}{b}$,

$u_{j}^{\prime}=\frac{1}{d}\left(j=1,2, \ldots, d^{\prime}-d\right), u_{d^{\prime}-d+1}^{\prime}=\frac{d^{\prime}}{d}$,

From equation (9) we have:

$\left(a-a^{\prime}\right)\left(b-b^{\prime}\right) h\left(\frac{1}{a b}, \frac{1}{c d}\right)+\left(b-b^{\prime}\right) h\left(\frac{a^{\prime}}{a b}, \frac{c^{\prime}}{c d}\right)+\left(a-a^{\prime}\right) h\left(\frac{b^{\prime}}{a b}, \frac{d^{\prime}}{c d}\right)+h\left(\frac{a^{\prime} b^{\prime}}{a b}, \frac{c^{\prime} d^{\prime}}{c d}\right)$

$=\left(\frac{-a_{2}}{a_{1}}\right)\left[\left(a-a^{\prime}\right) h\left(\frac{1}{a}, \frac{1}{c}\right)+h\left(\frac{a^{\prime}}{a}, \frac{c^{\prime}}{c}\right)\right]\left[\left(b-b^{\prime}\right) h\left(\frac{1}{b}, \frac{1}{d}\right)+h\left(\frac{b^{\prime}}{b}, \frac{d^{\prime}}{d}\right)\right]$

Taking $a^{\prime}=b^{\prime}=c^{\prime}=d^{\prime}=1$ in (11), we get:

$h\left(\frac{1}{a b}, \frac{1}{c d}\right)=\left(\frac{-a_{2}}{a_{1}}\right) h\left(\frac{1}{a}, \frac{1}{c}\right) h\left(\frac{1}{b}, \frac{1}{d}\right)$.

Taking $a^{\prime}=c^{\prime}=1$ in (11) and using (12), we have:

$h\left(\frac{b^{\prime}}{a b}, \frac{d^{\prime}}{c d}\right)=\left(\frac{-a_{2}}{a_{1}}\right) h\left(\frac{1}{a}, \frac{1}{c}\right) h\left(\frac{b^{\prime}}{b}, \frac{d^{\prime}}{d}\right)$.

Again taking $b^{\prime}=d^{\prime}=1$ in (11) and using (12), we get:

$$
h\left(\frac{a^{\prime}}{a b}, \frac{c^{\prime}}{c d}\right)=\left(\frac{-a_{2}}{a_{1}}\right) h\left(\frac{1}{b}, \frac{1}{d}\right) h\left(\frac{a^{\prime}}{a}, \frac{c^{\prime}}{c}\right)
$$

Now (11) together with (12), (13) and (13) reduces to:

$h\left(\frac{a^{\prime} b^{\prime}}{a b}, \frac{c^{\prime} d^{\prime}}{c d}\right)=\left(\frac{-a_{2}}{a_{1}}\right) h\left(\frac{a^{\prime}}{a}, \frac{c^{\prime}}{c}\right) h\left(\frac{b^{\prime}}{b}, \frac{d^{\prime}}{d}\right)$

Putting $\frac{a^{\prime}}{a}=p, \frac{c^{\prime}}{c}=u, \frac{b^{\prime}}{b}=p^{\prime}, \frac{d^{\prime}}{d}=u^{\prime}$ in (15), we get the required results (10).

Next we obtain the general solution of (10).

Lemma2.3. One of the general continuous solution of equation (10) is given by:

$h(p, u)=\left(\frac{-a_{1}}{a_{2}}\right)\left(\frac{p^{\mu} u^{v}}{p u}\right)^{1 / \mu}$, where $\mu \neq 0, v \neq 0$

and $h(p, u)=0$

Proof: Taking $g(p, u)=\left(\frac{-a_{2}}{a_{1}}\right) h(p, u)$ in (10), we have:

$g\left(p p^{\prime}, u u^{\prime}\right)=g(p, u) g\left(p^{\prime}, u^{\prime}\right)$

The most general continuous solution of (18) (refer to [13]) is given by: 
$g(p, u)=\left(\frac{p^{\mu} u^{v}}{p u}\right)^{1 / \mu}, \mu \neq 0$ and $v \neq 0$

and

$g(p, u)=\mathbf{0}$

On substituting $g(p, u)=\left(\frac{-a_{2}}{a_{1}}\right) h(p, u)$ in (19) and (20) we get (16) and (17) respectively. This proves the lemma 2.3 for all rationals $p \in] 0,1[$ and $u>0$, However, by continuity, it holds for all reals $p \in] 0,1[$ and $u>0$.

Theorem2.1. The measure (8) can be determined by the axiom 2.1 to 2.4 .

Proof: Substituting the solution (16) in axiom 2.1 we have:

$G_{n}(P ; U)=a_{1}\left[1-\sum_{i=1}^{n}\left(\frac{p_{i}^{\mu} u_{i}^{v}}{p_{i} u_{i}}\right)^{1 / \mu}\right], \quad \mu \nu \neq 0$

Taking $p_{i}=\frac{1}{n}$ and $u_{i}=u$ for each $\mathrm{i}$ in (21) we have:

$G_{n}\left(\frac{1}{n}, \ldots, \frac{1}{n}, u, \ldots, u\right)=a_{1}\left(1-n^{\frac{1-\mu}{v}} u^{\frac{v-1}{v}}\right), \quad n=2,3, \ldots$,

Axiom (2.4) together with (22) gives:

$a_{1}\left(1-n^{\frac{1-\mu}{\mu}} u^{\frac{v-1}{v}}\right)=\frac{R}{R-1}\left[1-n^{\frac{1-R}{R}}\right]$

It implies

$a_{1}=\frac{R}{R-1}, \quad \mu=R, \quad v=1$

Putting these values in (21) we have

$$
\begin{gathered}
G_{n}(P ; U)=\frac{R}{R-1}\left[1-\left(\frac{\sum_{i=1}^{n} u_{i} p_{i}{ }^{R}}{\sum_{i=1}^{n} u_{i} p_{i}}\right)^{1 / R}\right] \\
=H_{R}(P ; U)
\end{gathered}
$$

Hence this completes the proof of theorem 2.1.

Particular cases:

(a) When utilities are ignored i.e. $u_{i}=1$ for each i, (8) reduces to (1).

(b) Further $R \rightarrow 1,(1)$ reduces to Shannon's entropy [13].

\section{Properties of 'useful' R-norm Information Measure}

The 'useful' R-norm information measure $H_{R}(P ; U)$ satisfies the following properties:

Property 3.1. $H_{R}(P ; U)$ is symmetric function of their arguments provided that the permutation of $p_{i}^{\prime} s$ and $u_{i}^{\prime} s$ are taken together.

$H_{R}\left(p_{1}, p_{2}, \ldots, p_{n-1}, p_{n} ; u_{1}, u_{2}, \ldots u_{n-1}, u_{n}\right)=H_{R}\left(p_{n}, p_{1}, p_{2}, \ldots, p_{n-1} ; u_{n}, u_{1}, u_{2}, \ldots, u_{n-1}\right)$

Property 3.2. $H_{R}\left(\frac{1}{8}, \frac{1}{8} ; 1,1\right)=1$

Proof: $H_{R}(P ; U)=\frac{R}{R-1}\left[1-\left(\frac{\sum u_{i} p_{i}{ }^{R}}{\sum u_{i} p_{i}}\right)^{1 / R}\right]$

for $i=1,2$

$H_{R}(P ; U)=\frac{R}{R-1}\left[1-\left(\frac{u_{1} p_{1}^{R}}{u_{1} p_{1}}+\frac{u_{2} p_{2}{ }^{R}}{u_{2} p_{2}}\right)^{1 / R}\right]$ 
Taking $p_{1}=\frac{1}{8}, \quad p_{2}=\frac{1}{8}, \quad u_{1}=1, \quad u_{2}=1$ and $R=2$

$H_{R}\left(\frac{1}{8}, \frac{1}{8} ; 1,1\right)=\frac{2}{1}\left[1-\left\{\frac{\left(\frac{1}{8}\right)^{2}}{\frac{1}{8}}+\frac{\left(\frac{1}{8}\right)^{2}}{\frac{1}{8}}\right\}^{1 / 2}\right]=1$

Property 3.3. Addition of two events whose probability of occurrence is zero or utility is zero has no effect on useful information, i.e.

$H_{R}\left(p_{1}, p_{2}, \ldots, p_{n}, \mathbf{0} ; u_{1} u_{2}, \ldots u_{n+1}\right)=H_{R}\left(p_{1}, p_{2}, \ldots, p_{n} ; u_{1}, u_{2}, \ldots, u_{n}\right)=H_{n}\left(p_{1}, p_{2}, \ldots, p_{n+1} ; u_{1}, u_{2}\right.$

Proof: Let us consider

$$
\begin{aligned}
& H_{R}\left(p_{1}, p_{2} \ldots, p_{n}, 0 ; u_{1}, u_{2}, \ldots, u_{n+1}\right)==\frac{R}{R-1}\left[1-\left\{\frac{u_{1} p_{1}{ }^{R}}{u_{1} p_{1}}+\frac{u_{2} p_{2}{ }^{R}}{u_{2} p_{2}}+\ldots+\frac{u_{n} p_{n}{ }^{R}}{u_{n} p_{n}}+\ldots+\frac{0^{R} \cdot u_{n+1}}{0 . u_{n+1}}\right\}^{1 / R}\right] \\
& \quad=H_{R}(P ; U)
\end{aligned}
$$

Similarly we can prove that

$H_{n}\left(p_{1}, p_{2}, \ldots, p_{n}, p_{n+1} ; u_{1}, u_{2}, \ldots, u_{n}, u_{n+1}\right)=H_{R}(P ; U)$

Property 3.4. $H_{R}(P ; U)$ satisfies the non-additivity of the following form:

$$
H_{R}(P * Q ; U * V)=H_{R}(P ; U)+H_{R}(Q ; V)-\frac{R-1}{R} H_{R}(P ; U) H_{R}(Q ; V)
$$

where $P * Q=\left(p_{1} q_{1}, \ldots, p_{1} q_{m}, p_{2} q_{1}, \ldots, p_{2} q_{m}, p_{n} q_{1}, \ldots, p_{n} q_{m}\right)$, and

$U * V=\left(u_{1} v_{1}, \ldots, u_{1} v_{m}, u_{2} v_{1}, \ldots, u_{2} v_{m}, u_{n} v_{1}, \ldots, u_{n} v_{m}\right)$

Proof: R.H.S $=H_{R}(P ; U)+H_{R}(Q ; V)-\frac{R}{R-1} H_{R}(P ; U) H_{R}(Q ; V)$

$$
\begin{aligned}
& \left.=\frac{R}{R-1}\left[1-\left(\frac{\sum u_{i} p_{i}{ }^{R}}{\sum u_{i} p_{i}}\right)^{1 / R}\right]+\frac{R}{R-1}\left[1-\left(\frac{\sum v_{j} q_{j}{ }^{R}}{\sum v_{j} q_{j}}\right)^{1 / R}\right]-\frac{R}{R-1}\left[1-\left(\frac{\sum u_{i} p_{i}{ }^{R}}{\sum u_{i} p_{i}}\right)^{1 / R}\right] 1-\left(\frac{\sum v_{j} q_{j}{ }^{R}}{\sum v_{j} q_{j}}\right)^{1 / R}\right] \\
& =\frac{R}{R-1}\left[1-\left(\frac{\sum u_{i} p_{i}{ }^{R}}{\sum u p_{i}}\right)^{1 / R}+1-\left(\frac{\sum v_{j} q_{j}{ }^{R}}{\sum v_{j} q_{j}}\right)^{1 / R}-1+\left(\frac{\sum u_{i} p_{i}{ }^{R}}{\sum u_{I} p_{i}}\right)^{1 / R}+\left(\frac{\sum q_{j} v_{j}{ }^{1}}{\sum q_{j} v_{j}}\right)^{1 / R}-\left(\frac{\sum u_{i} p_{i}^{R}}{\sum u_{I} p_{i}}\right)^{1 / R}\left(\frac{\sum q_{j} v_{j}{ }^{R}}{\sum q_{j} v_{j}}\right)^{1 / R}\right] \\
& =\frac{R}{R-1}\left[1-\left(\frac{\sum u_{i} p_{i}{ }^{R}}{\sum u_{I} p_{i}}\right)^{1 / R}\left(\frac{\sum q_{j} v_{j}{ }^{R}}{\sum q_{j} v_{j}}\right)^{1 / R}\right]=\frac{R}{R-1}\left[1-\left(\frac{\sum_{i=1}^{n} \sum_{j=1}^{m} u_{i} v_{j}\left(p_{i} q_{j}\right)^{R}}{\sum_{i=1}^{n} \sum_{j=1}^{m} u_{i} v_{j} p_{i} q_{j}}\right)^{1 / R}\right] \\
& =H_{R}(P * Q ; U * V)=\text { L.H.S. }
\end{aligned}
$$

Property 3.5. Let $A_{i}, A_{j}$ be two events having probabilities $p_{i}, p_{j}$ and utilities $u_{i}, u_{j}$ respectively, then we define the utility $u$ of the compound event $A_{i} \cap A_{j}$ as:

$$
u\left(A_{i} \cap A_{j}\right)=\frac{u_{i} p_{i}+u_{j} p_{j}}{p_{i}+p_{j}}
$$

Theorem 3.1 Under the composition law (23), the following holds:

${ }_{n+1} H_{R}\left(p_{1}, p_{2}, \ldots, p_{n-1}, p^{\prime}, p^{\prime \prime} ; u_{1} u_{2}, \ldots, u_{n-1}, u^{\prime}, u^{\prime \prime}\right)={ }_{n} H_{R}(P, U)+\left(p^{\prime}+p^{\prime \prime}\right) H_{R}\left(\frac{p^{\prime}}{p^{\prime}+p^{\prime \prime}}, \frac{p^{\prime \prime}}{p^{\prime}+p^{\prime \prime}} ; u^{\prime}, u^{\prime \prime}\right)$,

where $p_{n}=p^{\prime}+p^{\prime \prime}$ and $u_{n}=\frac{p^{\prime} u^{\prime}+p^{\prime \prime} u^{\prime \prime}}{p^{\prime}+p^{\prime \prime}}$

Proof: ${ }_{n+1} H_{R}\left(p_{1}, p_{2}, \ldots, p_{n-1}, p^{\prime}, p^{\prime \prime} ; u_{1}, u_{2}, \ldots, u_{n-1}, u^{\prime}, u^{\prime \prime}\right)$ 


$$
\begin{aligned}
& ={ }_{n} H_{R}\left(p_{1}, p_{2}, \ldots, p_{n-1} ; u_{1}, u_{2} \ldots, u_{n-1}\right)+\frac{R}{R-1}\left[1-\left\{\frac{u^{\prime} p^{\prime R}}{u^{\prime} p^{\prime}}+\frac{u^{\prime \prime} p^{\prime \prime}}{u^{\prime \prime} p^{\prime \prime}}\right\}^{1 / R}\right] \\
& ={ }_{n} H_{R}+\frac{R}{R-1}\left[\left(p^{\prime}+p^{\prime \prime}\right)-\left\{\frac{u^{\prime}\left(\frac{p^{\prime}}{p^{\prime}+p^{\prime \prime}}\right)^{R}+u^{\prime \prime}\left(\frac{p^{\prime \prime}}{p^{\prime}+p^{\prime \prime}}\right)^{R}}{u^{\prime}\left(\frac{p^{\prime}}{p^{\prime}+p^{\prime \prime}}\right)+u^{\prime \prime}\left(\frac{p^{\prime \prime}}{p^{\prime}+p^{\prime \prime}}\right)}\right\}^{1 / R}\left(p^{\prime}+p^{\prime \prime}\right)\right] \\
& ={ }_{n} H_{R}+\left(p^{\prime}+p^{\prime \prime}\right) H_{R}\left(\frac{p^{\prime}}{p^{\prime}+p^{\prime \prime}}, \frac{p^{\prime \prime}}{p^{\prime}+p^{\prime \prime}} ; u^{\prime}, u^{\prime \prime}\right)
\end{aligned}
$$

This completes the proof of theorem 3.1.

\section{Conclusion}

$\mathrm{R}$ - norm information measure is defined and characterized when the probability distribution $\mathrm{P}$ belong to R- norm vector space. This is a new addition to the family of generalized information measures.

In present paper we have considered that physical system has qualitative characterization in addition to quantitative and have defined and characterized a new measure R-norm information measure. This measure can further be generalized in many ways and can be applied in source coding when source symbols have utility also in addition to probability of occurrence.

\section{References}

[1]. D. E. Boekee, and J.C.A. Van Der Lubbe, The R-norm Information measure, Information and control, 45, 1980,136 -155.

[2]. C.E. Shannon, A mathematical theory of communication. Bell System Technology Journal, 27, 1948, $379-423$.

[3]. A. Renyi, On measure of entropy and information, Selected paper of Alfored Renyi, 2, 1976, 565-580

[4]. J.Havrda, and F. Charvat, Quantification of classification processes concept of Structural $\boldsymbol{\alpha}$ - entropy. Kybernetika, 3, 1967, 3035 .

[5]. Z. Daroczy, Generalized information function, Information and control, 16, 1970 36-51.

[6]. D.S. Hooda, and Anant Ram, Characterization of the generalized R-norm entropy, Caribbean journal of Mathematical and Computer science, 8, 2002 18-31.

[7]. S.Arimoto, Information theoretical consideration on estimation proems. Information and control, 19, 1971, $181-199$.

[8]. D.S. Hooda, and D.k. Sharma, Generalized R-norm information measures, Journal of Application Math statistics \& Informatics, 4(2), 2008 153-168.

[9]. D.S. Hooda, Saxena Sonali, and D.k. Sharma, A Generalized R-norm entropy and coding theorem. International journal of Mathematical Sciences and Engineering Applications, 5, 2011, 385-393.

[10]. G. Longo, Quantitative-Qualitative Measure of Information, Springer-Verlag, NewYork, 1972.

[11]. M. Belis, and Guiasu, A quantitative - qualitative measure of information in Cybernetics, System IEEE Trans. Information Theory, IT 14, 1968, 593-594.

[12]. U.S. Bhaker, and D.S. Hooda, Mean value characterization of 'useful' information measures, Tamkang Journal of Mathematics, 24, 1993, 383-394

[13]. J. Aczel, Lectures on functional equations and their applications (Academic Press, New York 1966). 